\title{
Idiopathic Hypertrophic Dorsal Pachymeningitis - Imaging Features
}

\author{
Krishna Kumar $^{1}$, R. Rajalakshmi Preethi ${ }^{2}$ \\ ${ }^{1}$ Professor \& Head, Department of Radiodiagnosis. Velammal Medical College \& Research Institute, Velammal Village, Tuticorin Ring Road, Anuppanadi, \\ Madurai.Tamilnadu-625009, ${ }^{2}$ Associate Professor, Department of Radiodiagnosis, Velammal Medical College \& Research Institute, Velammal Village, Tuticorin \\ Ring Road, Anuppanadi, Madurai.Tamilnadu-625009.
}

\begin{abstract}
Idiopathic Hypertrophic Spinal Pachymeningitis (IHSP) is a rare disease causing chronic inflammatory hypertrophy of the dura mater. It may be idiopathic or secondary to other diseases. It is an extremely rare cause of spinal cord compression. This process can be present throughout the entire spine but is commonly reported in the cervical and dorsal spine. IHSP is a diagnosis of exclusion since it is associated with trauma, infection, and autoimmune diseases.

We describe a rare case of Idiopathic Hypertrophic Dorsal Pachymeningitis (IHDP) in a 42 -year -old woman presenting with progressive weakness of both lower limbs for the past 2 years and outline the important clinical and radiographic features of the disease.
\end{abstract}

Keywords: Dural thickening, Hypertrophic Spinal Pachymeningitis, Idiopathic Hypertrophic Spinal Pachymeningitis, Paraparesis, Spinal Cord Compression.

Corresponding Author: Dr. Krishna Kumar, M, MD, DMRD. Professor \& Head, Department of Radiodiagnosis. Velammal Medical College \& Research Institute, Velammal Village, Tuticorin Ring Road, Anuppanadi, Madurai.Tamilnadu-625009.

Received: November 2018

Accepted: December 2018

\section{Introduction}

Idiopathic Hypertrophic Spinal pachymeningitis (IHSP) was first described in 1869 by Charcot and Joffroy. ${ }^{[1]}$ Idiopathic hypertrophic pachymeningitis (IHP) is a chronic progressive diffuse inflammatory fibrosis of the dura mater. ${ }^{[2-5]}$ This rare disorder is usually found intracranially and is a diagnosis of exclusion because meningioma, lymphoma, tuberculosis, sarcoidosis and other diseases may present in a very similar fashion. ${ }^{[2-6]}$ The spinal form is extremely rare, with few described cases in the literature. It occurs alone, usually involving the cervical and thoracic dura or as a craniospinal form. ${ }^{[2,7]}$ According to the location of the lesion, it might induce neurologic deficits by compression of spinal cord and nerve root.

IHSP commonly presents in the 6th and 7th decades and can be either solitary or multiple, and diffuse or nodular. ${ }^{[8]}$ The natural history is progression from local pain to radiculopathy and an eventual myelopathy. The etiology is unknown, but trauma, infection, tumors and various autoimmune diseases have been implicated. ${ }^{[9]}$

The aim of this study was to demonstrate Imaging features of Idiopathic Hypertrophic Dorsal Pachymeningitis (IHDP), in a 42 years old woman who presented with difficulty in walking \& progressive weakness of both lower limbs, caused due to spinal cord compression.

\section{Case Report}

A 42-year-old woman presented for evaluation of difficulty in walking, insidious in onset followed by gradual progressive weakness of lower extremities.

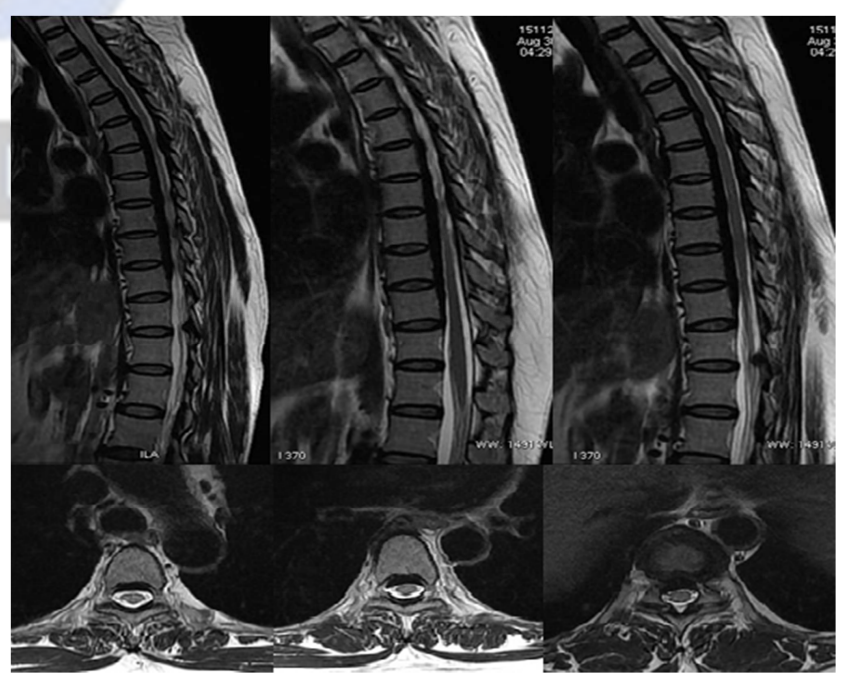

Figure 1: Sagittal (A) and Axial (B) MRI T2-weighted images. (A) Sagittal (a-c) MRI T2-weighted image showing a dural lesion of low signal intensity within the spinal canal at levels T2 to T11 extending anteriorly. (B) Axial (a-c) MRI T2weighted image at T2 and T11 level showing a dural mass in the anterior aspect of the spinal canal.

There were no exacerbating or alleviating factors. She had no history of trauma, fever, weight change, or night sweats. Her past medical history was noncontributory. On examination, there was hyperreflexia and clonus of her lower extremities, with no clear sensory level to pinprick testing. Otherwise, her general physical examination results 
Thumar d Preethi; Idiapathic Ftypertrophic Darsal Pachymeningitis

were normal.

The routine Blood tests, Radiographs of the chest \& spine , Serum angiotensin converting enzyme levels, Erythrocyte sedimentation rate, Cerebrospinal fluid routine examination, Adenosine deaminase level and Polymerase chain reaction for tuberculosis, and Venereal Disease Research Laboratory fluorescent treponemal antibody absorption were normal. MRI of the brain showed no meningeal involvement and was grossly normal [Figure 1, 2, 3, 4].

She underwent magnetic resonance imaging (MRI) and computed tomographic (CT) imaging of her spine. Patient refused to undergo contrast enhanced MRI.
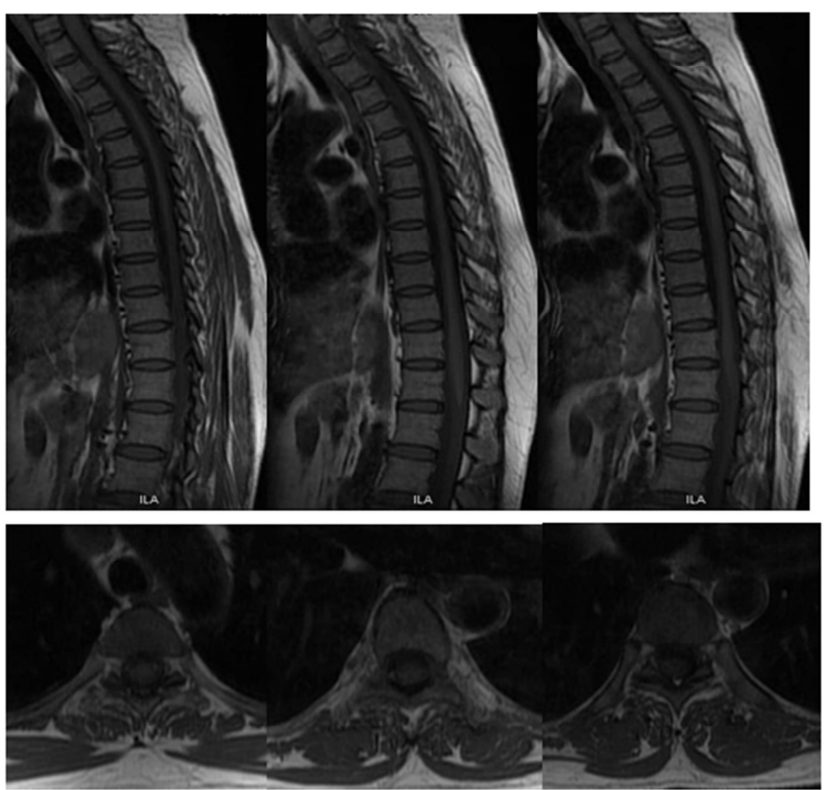

Figure 2: Sagittal (A) and Axial (B) MRI T1-weighted images. (A) Sagittal (a-c) MRI T1-weighted image showing a shows a hypointense ventral dural based extramedullary mass spanning vertebral levels T2 till T11. (B) Axial (a-c) MRI T1weighted image at $\mathrm{T} 2$ to $\mathrm{T} 11$ level showing a dural mass in the anterior aspect of the spinal canal.

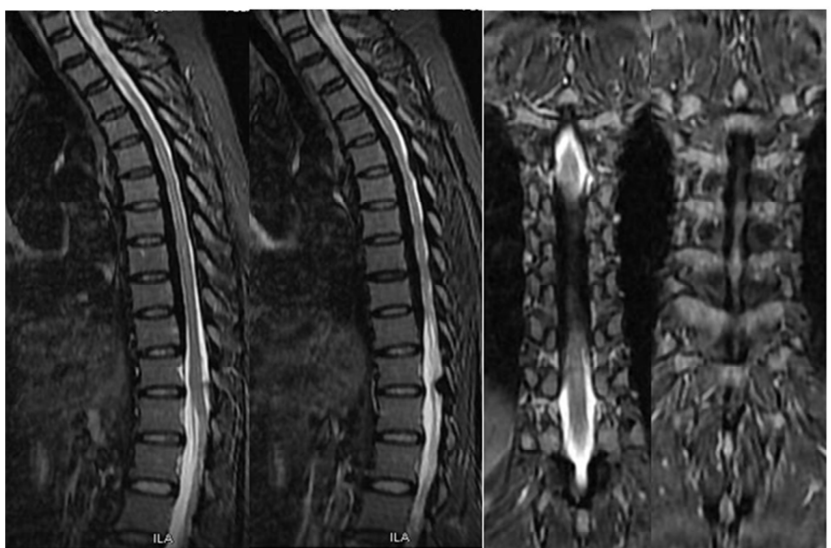

Figure 3: Sagittal (A) and Coronal (B) MRI STIR- images. (A) Sagittal $(a, b)$ MRI STIR- image showing a dural lesion of low signal intensity within the spinal canal at levels T2 to T11 extending anteriorly. (B) Coronal (a, b) MRI STIR- image at T2 till T11 level showing a hypointense dural mass in the anterior aspect of the spinal canal. No evidence of cord edema or myelomalacia.

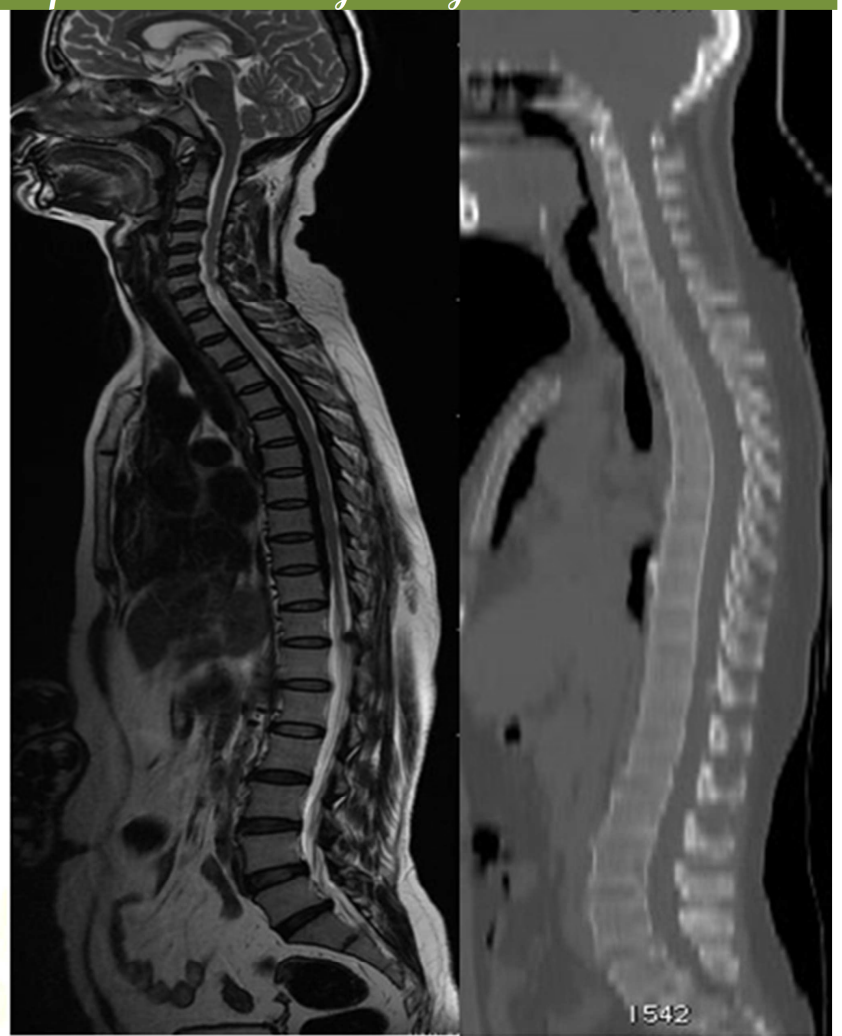

Figure 4. Sagittal (A) Pasted MRI Image and Sagittal (B) Reconstructed CT image. (A) Sagittal Pasted T2W MRI Image showing a dural lesion of low signal intensity within the spinal canal at levels T2 to T11 extending anteriorly. (B) Sagittal Reconstructed CT image at $\mathrm{T} 2$ to $\mathrm{T} 11$ level showing normal spinal canal without any evidence of calcification.

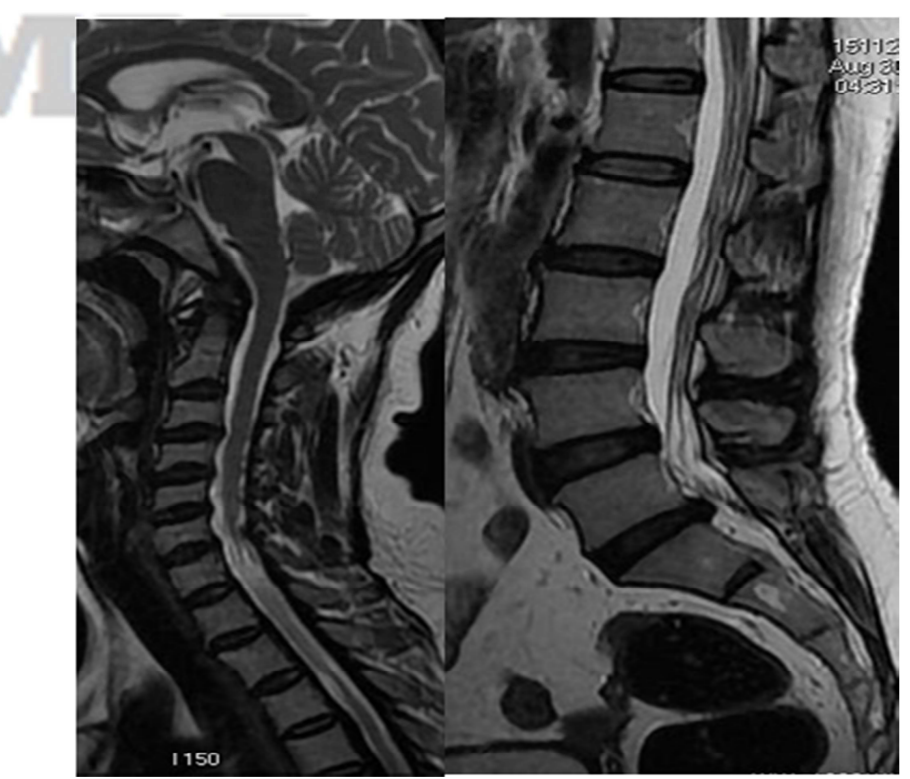

Figure 5. Sagittal (A) Cervical and Sagittal (B) Lumbar MRI T2W- images. (A) Sagittal Cervical and (B) Sagittal Lumbar T2 weighted MRI image showing no evidence of hypointense dural lesion within the spinal canal.

In our case we have not been able to find any causative or related diseases and believe it to be an idiopathic 
hypertrophic dorsal pachymeningitis. Considering the chronic progressive neurologic symptoms and characteristic appearance at MR imaging with absence of calcifications at CT imaging, the diagnosis of IHDP was made [Figure 4].

The patient was advised surgery, but she refused to undergo surgery and she is on regular follow up. However, given the preservation of strength and sensation in her legs, surgery was deemed to be of limited benefit. One-year follow-up of the patient has still not revealed any signs or symptoms of associated diseases. The symptoms and imaging findings remained the same.

\section{Discussion}

Hypertrophic Spinal Pachymeningitis (HSP) is a subtype of hypertrophic pachymeningitis, a rare disease characterized by localized or diffuse hypertrophic thickening of the dura matter. IHSP is a chronic inflammatory fibrosis of the dura mater and is an extremely rare cause of nerve root and spinal cord compression. ${ }^{[2]}$ In IHSP the male to female rate is 3:2 and age distribution range from 15 to 77 years with a mean age of 46 years. ${ }^{[10]}$

The Aetiology of hypertrophic pachymeningitis is unknown, but several causative factors have been recognized, among them Wegener's granulomatosis, Mixed Connective Tissue Disease, Systemic Lupus Erythematosus, Sarcoidosis, Multifocal Fibrosclerosis, Orbital Pseudotumor, Rheumatoid arthritis, Carcinomatosis, Metabolic diseases, Trauma, Toxins, Thrombophlebitis, adjacent to ear or sinus infection, infections such as Syphilis, Tuberculosis, Fungi, HIV, HTLV-1 and Meningococcal meningitis and intrathecal steroid deposition. ${ }^{[7,11-13]}$ Therefore extensive work up is required to rule out infectious, neoplastic, autoimmune, traumatic, toxic or metabolic cause to diagnose a patient as idiopathic hypertrophic pachymeningitis. In most cases a cause cannot be identified, leading to an idiopathic designation.

It has been proposed that hypertrophic spinal pachymeningitis (HSP) should be considered in the differential diagnosis for patients with spinal cord compression and radicular pain in more than three spinal levels. ${ }^{[12]}$

The classic disease course, first described by Charcot and Joffrey, ${ }^{[14]}$ in 3 stages: the first stage characterized by pain (either local or radicular), the second stage has clinical signs of nerve root compression, and the third stage is characterized by spinal cord compression.

The presentation of IHSP can vary from radicular symptoms to progressive myelopathy and involvement of sphincters in the later stages. Patients do not necessarily follow this pattern, however, and they can present with any combination of pain, radiculopathy, or myelopathy.

The cranium and spinal cord can be involved concomitantly, ${ }^{[15]}$ however, in our case there was an isolated dorsal spinal cord involvement.

On MRI, IHSP has been showed as dura-based mass of low signal intensity on T1- and T2-weighted images extending over multiple vertebral levels. ${ }^{[13,16]}$ In addition, predominantly peripheral enhancement pattern is a highly suggestive of IHSP. This pattern represents more intense enhancement in a peripheral zone of active inflammation than in a central zone of fibrosis. ${ }^{[13,16]}$ Linear dural enhancement pattern was known to have better response to steroid therapy than those with nodular pattern because of its less fibrosis and more vascularity. ${ }^{[13,15,16]}$

Pathologically, the dura is hyalinised, thickened and chronically inflamed with presence of granulation tissue formation. There is no evidence of calcification, malignancy or cyst formation.

At Histologic analysis, HSP shows active inflammation in the lesion periphery, with a central zone of dense fibrosis. ${ }^{[4]}$ Radiographic and Pathological confirmation, along with exclusion of other known etiologies, are the cornerstone for its diagnosis.

There are several Differential diagnostic considerations for an extramedullary mass causing cord displacement, which include-Epidural hematoma, Epidural abscess, Ossification of the posterior longitudinal ligament, Meningioma \& Epidural venous plexus engorgement. Other systemic or inflammatory processes, such as Sarcoidosis, Extramedullary hematopoiesis, or Myofibroblastic tumor, are rarer possibilities. ${ }^{[17]}$

At present, decompressive surgery for symptomatic compressive lesions and corticosteroid therapy for the inflammatory cascade remain the mainstay of treatment. Our patient refused to undergo surgery and she was put on corticosteroid therapy. The clinical course of IHSP may follow 1 of 3 patterns; sustained remission, relapse with corticosteroid resistance or relapse with corticosteroid dependence. ${ }^{[18]}$

In our case, we could not find any predisposing illnesses such as infectious diseases or autoimmune diseases in spite of the through investigations.

On one-year follow-up of the patient, the symptoms and imaging findings remained the same.

\section{Conclusion}

Although IHDP is a rare disorder, the clinical and radiological findings are characteristic. Unaccountable motor weakness and thickened dura as shown by spinal MRI probably lead to a diagnosis. Hypertrophic Spinal pachymeningitis should be considered as a differential diagnosis in the evaluation of spinal cord compression by an epidural mass. Decompressive surgery for symptomatic compressive lesions and corticosteroid therapy for the inflammatory cascade remain the mainstay of treatment.

\section{References}

1. Charcot JM, Joffroy A: Deux cas d'atrophie musculaire progressive avec lesions de la substance grise et des faisceaux anterolateraux de la molle epiniere. Arch Physiol Norm Pathol; 1869; 2:354-67: 744-69.

2. Kupersmith MJ, Martin V, Heller G, et al. Idiopathic hypertrophic pachymeningitis. Neurology 2004;62:686-94

3. Riku S, Kato S. Idiopathic hypertrophic pachymeningitis Neuropathology 2003;23:335-44.

4. Martin N, Masson C, Henin D, et al. Hypertrophic cranial pachymeningitis : assessment with $\mathrm{CT}$ and MR imaging. AJNR Am J Neuroradiol 1989;10:477-84. 
5. Lee YC, Chueng YC, Hsu SW, et al. Idiopathic hypertrophic cranial pachymeningitis: case report with 7 years of imaging follow-up. AJNR Am J Neuroradiol 2003;24:119-23.

6. Parney IF, Johnson ES, Allen PBR. Idiopathic cranial hypertrophic pachymeningitis responsive to antituberculous therapy: a case report. Neurosurgery 1997;41:965-71.

7. Ashkenazi F, Constantini S, Pappo O, et al. Hypertrophic spinal pachymeningitis: report of two cases and review of the literature. Neurosurgery 1991;28:730-32.

8. Rosenfeld JV, Kaye AH, Davis S, Gonzales M. Pachymeningitis cervicalis hypertrophica. Case report. J Neurosurg 1987;66:137-9.

9. Hassin GB. Histogenesis of cerebral hypertrophic pachymeningitis and its relation to syphilis. Am J Syphilis 1918;2:715.

10. Tsutsui M, Yasuda T, Kanamori M, Hori T, Kimura T: Long-term outcome of idiopathic hypertrophic thoracic pachymeningitis. Eur Spine J; May 2011; DOI 10.1007/s00586-011-1848-9

11. Bernat JL, Sadowsky CH, Vincent FM, Nordgren RE, Margolis G: Sclerosing spinal pachymeningitis. A complication of intratecal administration of Depo-Medrol for multiple sclerosis. J Neurol Neurosur PS; 1976; 39: 1124-28.

12. Takahashi H, Wada A, Yokoyama Y, Ishii M, Shibuya K, Suguro T: Idiopathic hypertrophic spinal pachymeningitis: a case report. JOS;

\section{0; 18(1): 113-7.}

13. Dumont AS, Clark AW, Sevick RJ, Myles ST: Idiopathic Hypertrophic Pachymeningitis: A Report of Two Patients and Review of the Literature. Can J Neurol Sci; 2000; 27: 333-40

14. Bucy PC, Freeman LW: Hypertrophic spinal pachymeningitis with special reference to appropriate surgical treatment. J Neurosurg; 1952; 9: $564-78$.

15. Botella C, Orozco M, Navarro J, Riesgo P. Idiopathic chronic hypertrophic craniocervical pachymeningitis: Case report. Neurosurgery 1994;35:1144-9.

16. Pai S, Welsh CT, Patel S, Rumboldt Z: Idiopathic Hypertrophic Spinal Pachymeningitis: Report of Two Cases with Typical MR Imaging Findings. AJNR; 2007; 28:590-2.

17. Jeon YK, Chang KH, Suh YL, Jung HW, Park SH. Inflammatory myofibroblastic tumor of the central nervous system: clinicopathologic analysis of 10 cases. J Neuropathol Exp Neurol 2005;64(3):254-259

18. Smucker JD, Ramme AJ, LeBlond RF, Bruch LA, Bakhshandehpour G: Hypertrophic Spinal Pachymeningitis With Thoracic Myelopathy The Initial Presentation of ANCA-related Systemic Vasculitis. J Spinal Disord Tech Volume; 2011; 24(8): 525-32.

Copyright: (C) the author(s), publisher. Asian Journal of Medical Radiological Research is an Official Publication of "Society for Health Care \& Research Development". It is an open-access article distributed under the terms of the Creative Commons Attribution Non-Commercial License, which permits unrestricted non-commercial use, distribution, and reproduction in any medium, provided the original work is properly cited.

How to cite this article: Kumar K, Preethi RR. Idiopathic Hypertrophic Dorsal Pachymeningitis - Imaging Features. Asian J. Med. Radiol. Res. 2018;6(2):04-07.

DOI: dx.doi.org/10.21276/ajmrr.2018.6.2.2

Source of Support: Nil, Conflict of Interest: None declared. 